\title{
Colloidal Gold. Part I
}

\author{
HISTORICAL AND PREPARATIVE ASPECTS, MORPHOLOGY AND STRUCTURE
}

\author{
John Turkevich \\ Department of Chemistry, Princeton University, Princeton, New Jersey, U.S.A.
}

\begin{abstract}
Colloidal gold bas a long history (1). Gold's lustre, chemical stability and high price fascinated many ancients. Its preparation to give beautiful ruby red 'solution' intrigued the alchemists. Unusual medical properties were ascribed to this 'potable gold' and hope was expressed that this may be the 'elixir of life.' This hope found a modest realization in the 'Danziger Gold', an alcoholic concoction containing finely-divided gold. Still available, its exbilarating effects are due not to the gold but to its alcobolic content. Gold ruby glass was manufactured at the end of the 16th century and the 'Purple of Cassius', prepared by the reduction of soluble gold salts by tin chloride, bas been known since the 17th century.
\end{abstract}

Scientific interest in colloidal systems arose in the middle of the 19th century. In 1857 Michael Faraday published a comprehensive account of the preparation and properties of colloidal gold (2). His preparation was stable for almost a hundred years, only to be destroyed in the bombardment of London during World War II. During the last quarter of the 19th century and in the beginning of the 20th, interest in colloidal gold broadened: Bredig developed hisarc method for its preparation (3), Zsigmondy invented the slitultramicroscope (4), Mie gave the theoretical explanation for the colour of colloidal gold (5), Schulze (6) and Hardy (7) studied its stability in the presence of ions of different valencies, Einstein (8) gave the theory of its Brownian motion and Smoluchowski (9) formulated the theory of the coagulation process. Medical applications were also pursued. Colloidal gold was, and is still, used for treatment of arthritis. A number of diseases were diagnosed by the interaction of colloidal gold with spinal fluids obtained from the patient.

After World War I interest in colloidal phenomena shifted from inorganic systems to high molecular weight organic substances, both polymers and biochemicals. Courses in many universities in colloidal chemistry were abandoned. Research in classical colloid chemistry was handicapped by the inability of the optical microscopes with their resolving power of $200 \mathrm{~nm}$ to investigate colloidal particles of $2.5 \mathrm{~nm}$ diameter.

The invention of the electron microscope at the start of World War II opened up the field of colloids for detailed study. The resolution of the electron microscope was $2 \mathrm{~nm}$ in 1945 and has decreased now in 1985 to $0.2 \mathrm{~nm}$. Individual heavy atoms can be visualized and atomic lattice spacings delineated. Pioneering investigations in colloid chemistry using this new tool were carried out under trying war conditions in Germany by Borries and Kausche (10) and by von Ardenne and Thiessen (11). A comprehensive investigation of colloidal gold using the electron mictoscope as the main tool was started in 1948 at Princeton University and the RCA Laboratories by the author and his colleagues $(12,13)$.

Work was also carried out at other laboratories in Japan by Takiyama (14), by Suito et al. (15) and in the United States by Morriss and Milligan (16).

\section{Preparation}

Two basic approaches are used in the preparation of colloidal gold solutions. One is the disintegration of metallic gold rods by an electric arc operating in a liquid medium (Bredig (2) method and its modification by Svedberg in (1)). The other more general approach is the synthesis of particles from gold salts using either appropriate reducing agents or radiation (ultrasonic (17), pulse and laser radiolysis (18)). Examination of many prepatations proposed in the literature showed that most of them had limited stability and a broad particle distribution (13). However, the reduction of chlorauric acid by sodium citrate at $100{ }^{\circ} \mathrm{C}$ produced a colloidal solution (standard citrate sol) which had excellent stability and 'uniform' particle size of $20 \mathrm{~nm}$ diameter. The electron mictoscope was used to determine the particle size distribution and thus to evaluate the 'uniformity' of the preparation (Figure 1). Its standard deviation from the mean of 12 per cent indicated that 62 per cent of the particles had diameters between 18 to $22.4 \mathrm{~nm}$. In addition, the particle size distribution curve had not a Gaussian shape but showed 'character', having a steep drop on the larger diameter side and a long tail on the smaller particle side.

Both the average diameter and the character of the distribution curve changed with preparative conditions - concentration, ratio of reactants, temperature (Figure 2). This was taken to indicate that the process of particle formation was chemical in nature and involved interplay of three steps - nucleation, growth and coagulation. 'Uniformity' of the particles in the standard citrate sol was due to a favourable juxtaposition of these three steps: nucleation took place and stopped, then growth took over and at the same time the particles attained a stability which prevented the widening of the distribution curve by coagulation. In order to understand the preparation process these three steps had to be studied individually.

\section{Growth}

The first step to be isolated and studied was that of growth. It had been previously found that whenchlorauric solution was treated at toom temperature with hydroxylamine hydrochloride, no colloid was produced unless proper nuclei were present. By inoculating such a growth medium with a controlled number of approptiate nuclei, 


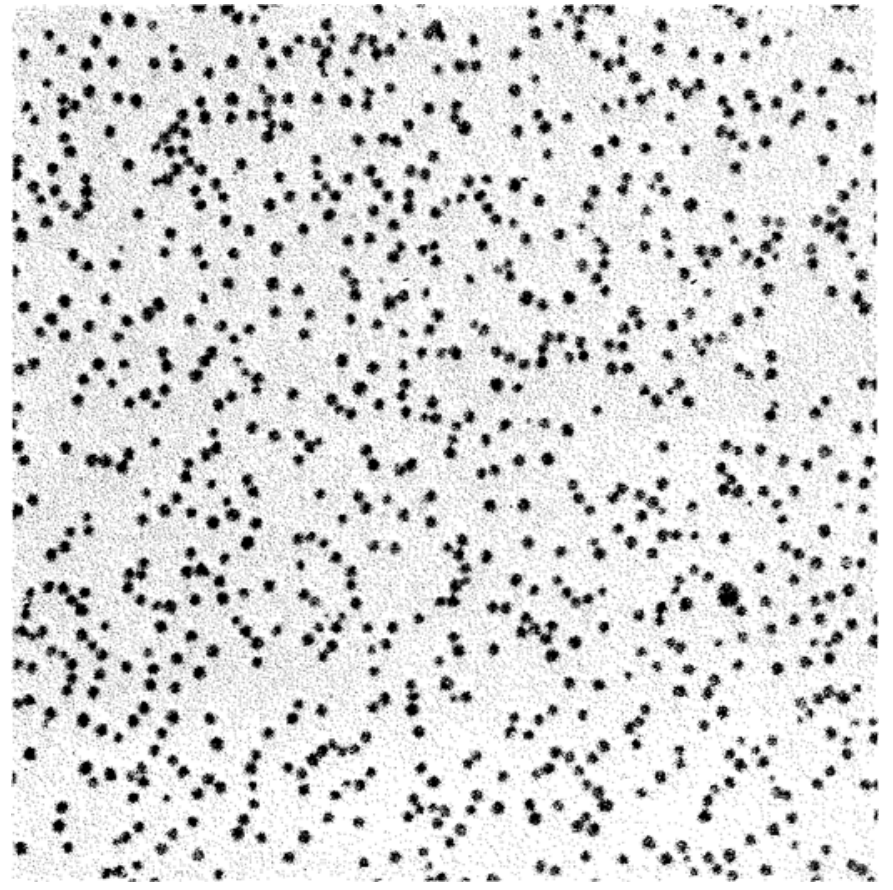

Fig. 1 Electron micrograph of standard citrate sol

$x 120000$

these could be made to grow to a desired size given by the following relation

$$
D_{f}=D_{n}\left(\frac{A u_{n}+A u_{g}}{A u_{n}}\right]^{1 / 3}
$$

where $D_{f}$ is the final diameter, $D_{n}$ is the diameter of the nucleus, $A u_{n}$ is the amount of gold in the nuclei, and $A u_{g}$ is the amount of gold in the growth medium. Thus to obtain $40 \mathrm{~nm}$ particles from $20 \mathrm{~nm}$ particles, one would use seven times the amount of gold in the growth medium as in the $20 \mathrm{~nm}$ nucleating gold sol. In this way a graded set of monodisperse colloidal gold solutions was obtained in which the average particle diameters were up to $120 \mathrm{~nm}$. This permitted the study of the effect of size on the properties of colloidal gold. Examination of the particle size distribution curves of such sols showed that while they broadened on growth, the standard deviation from the mean remained approximately constant at 10 percent. This was taken to indicate that the law of growth was given by

$$
\frac{\mathrm{dD}}{\mathrm{dt}}=\text { const. } \mathrm{D}
$$

where $D$ is the diameter of the gold particle. This law states that the larger is the particle the faster it grows, which was checked by studying the growth rate of particles of two different diameters. The law of growth also states that the smaller is the particle the slower is the growth. This implies that there is a particle size below which growth rate is negligible, which defines the size of a nucleus originally estimated at $4 \mathrm{~nm}$ and later found to be $1.0-2.0 \mathrm{~nm}$.

\section{Nucleation}

The author $e t a l$. (13) then proceeded to study in more detail the particle size distribution curve of the standard citrate sol. This 20 nm diameter sol was grown to produce sols with two average particle diameters, $40 \mathrm{~nm}$ and $80 \mathrm{~nm}$. A search was then made for the smallest particle in each of these preparations using an electron microscope with a resolution of $2 \mathrm{~nm}$. This was found to be of diameter $9 \mathrm{~nm}$ for the $40 \mathrm{~nm}$ sol and $18 \mathrm{~nm}$ for the $80 \mathrm{~nm}$ sol, indicating that the smallest particle that could grow, the nucleus, was about $4.5 \mathrm{~nm}$ in diameter. Since the particle size distribution curve is the result of a nucleation process followed by a growth process, the law of which had been determined, the rate curve for nucleation could be mathematically deduced from the particle size distribution curve. The rate curve (Figure 3) so obtained had an unusual form - an induction period, an autocatalytic rise in the number of nuclei, a slowing down of nuclei formation and finally, a cessation of the nucleation process, although the starting reagents were still present. It was further found that the

Fig. 2 (left) Particle size distribution curves of gold sol prepated at different concentrations

Fig. 3 (right) Nucleation rate curves for gold sols prepared at different concentrations - from Fig. 1 
induction period increased with decrease in temperature indicating the presence of a chemical reaction with an activation energy of 15 $\mathrm{Kcal} /$ mole.

Examination of the chemistry of the reducing agent suggested that the induction period might be due to the transformation of the citrate ion into acetone dicarboxylate ion. When the latter was used as a reducing agent, a different, more symmetrical particle size distribution curve was obtained. The nucleation curve deduced from it had no induction period but represented a unimolecular decomposition of a 'precursor' of the nucleus. This 'precursor', a complex formed from the gold ion and the acetone dicarboxylate ion, was isolated by removing the gold ion in the very early stages of preparation by means of an ion exchange resin. Electron microscopic examination revealed diffuse amorphous particles which on bombardment with an electron beam formed small, compact gold particles. Thus, gold nuclei do not form by reduction of individual gold ions to atoms which then collide to form, by fluctuations, a stable nucleus. Rather the nucleation process consists of a polymerization step. When a critical mass is attained, a reduction to metal patticle takes place. The nature of the polymerization step to form the 'precursor' may vary. In acid and neutral solution it is the formation of gold organic polymer, in alkaline solution a polymerization of gold hydroxide may take place (19). The unimolecular redox decomposition of the organic gold polymer or the facile reduction of the gold hydroxide polymer takes place when the degree of polymerization is sufficiently great to produce a stable gold particle, a particle whose cohesive lattice energy is greater than the disruptive sufface energy. The latter may be affected by adsorbed molecules. For nucleation in acid and neutral solutions, the reducing agent must have at least two reactive groups to bring about polymerization (such as citrate, hydroquinone, acetone carboxylic acid). On the other hand, for pure growth, the solution must be neutral or acidic to avoid formation of gold hydroxide and the reducing agent should have one functional group (hydroxylamine hydrochloride, hydrogen peroxide). Once a sufficient number of nuclei is formed, the growth process takes over. This is a consequence of the following: the growth process is a one-step autocatalytic process catalyzed by the nucleus while the nucleus formation is a multi-step process dependent on polymerization. Turkevich etal. (13) concluded that the nucleus was $4.5 \mathrm{~nm}$ in diameter. Subsequently Mortiss and Milligan (16) studying the effect of traces of copper on the nature of gold sol produced by hydrogen peroxide, found particles as small as $1.0 \mathrm{~nm}$. Uyeda and his collaborators (15) concluded that the nuclei had a diameter of 1.0 to $2.0 \mathrm{~nm}$ and that these nuclei coalesced into simple and multiple twins before growth took place. This viewpoint is confirmed by high resolution electron micrographs which show that standard citrate sol particles are not single crystals. In recent years there has been considerable activity in the synthesis and characterization of gold cluster compounds (20) and it would be of interest to see whether these could serve as nuclei for gold colloid particles.

\section{Coagulation}

The coagulation of particles is an important step in colloid preparation. Its control during the preparation process determines the shape, structure and the size distribution of the particles. Once the preparation is complete, its absence ensures the stability of the sol. On the other hand the nature of the coagulation process of a prepared sol determines the morphology of the coagulum.

In the standard citrate sol the particles have a negative charge due to the strong adsorption of the citrate ion to form what is known as the Stern layer on the colloidal particle. As we shall see later, this determines to the first approximation the stability of the colloid. It was originally proposed (13) that the $20 \mathrm{~nm}$ diameter particle of the standard citrate sol was formed by pure growth from a $4.5 \mathrm{~nm}$ diameter nucleus. In view of subsequent work, this view must be modified. The $20 \mathrm{~nm}$ diameter particle is not a single crystal but multiple twin, often with flat sides. Furthermore, if growth is repressed during the preparation process $(21,22)$, plate-like structures are produced (Figure 4). These may be smooth flat plates or plates with spiral steps on their surfaces. The spiral often has a hole which passes through the centre of the plate. In the mechanism for the synthesis of the $20 \mathrm{~nm}$ diameter particle of the standard citrate sol we must postulate the production of small atomic clusters from the precursor which then may aggregate to form multiple twins (Figure 5).

These adsorb sufficient citrate to prevent further aggregation and thus serve as nuclei for growth to form the $20 \mathrm{~nm}$ diameter gold particles. In the mechanism for plate formation, the small nuclei aggregate to form linear chains. When the linear aggregates are sufficiently long, lateral forces produce rafts which ultimately change into flat smooth plates. Branching undoubtedly hinders the general plate formation. However, if the branching produces a chain spiral whose end overlaps some part of the chain, a spiral with a hole in the centre becomes a very favourable 'nucleus' for growth. Small nuclei adhere to the sides of the spiral and the spiral is not destroyed in this growth process. The height of the steps is determined by the diameter of the nuclei adhering to the spiral (Figure 4).

Since its introduction in 1951 by the author, Stevenson and Hillier, the standard citrate gold sol has found wide acceptance as a marker in electron microscopy and modifications of its preparation have been proposed by a number of workers $(23,24)$.

\section{Properties}

In this section we will discuss the morphology and structure characteristics of colloidal gold particles and the investigation of these by electron microscopy. The second part of this review to be published in the October 1985 issue of Gold Bulletin will describe other properties of these particles.

\section{Morphology}

Electron microscopic investigation has shown that colloidal gold sols studied had particles with diameters ranging from 1.0 to 160 


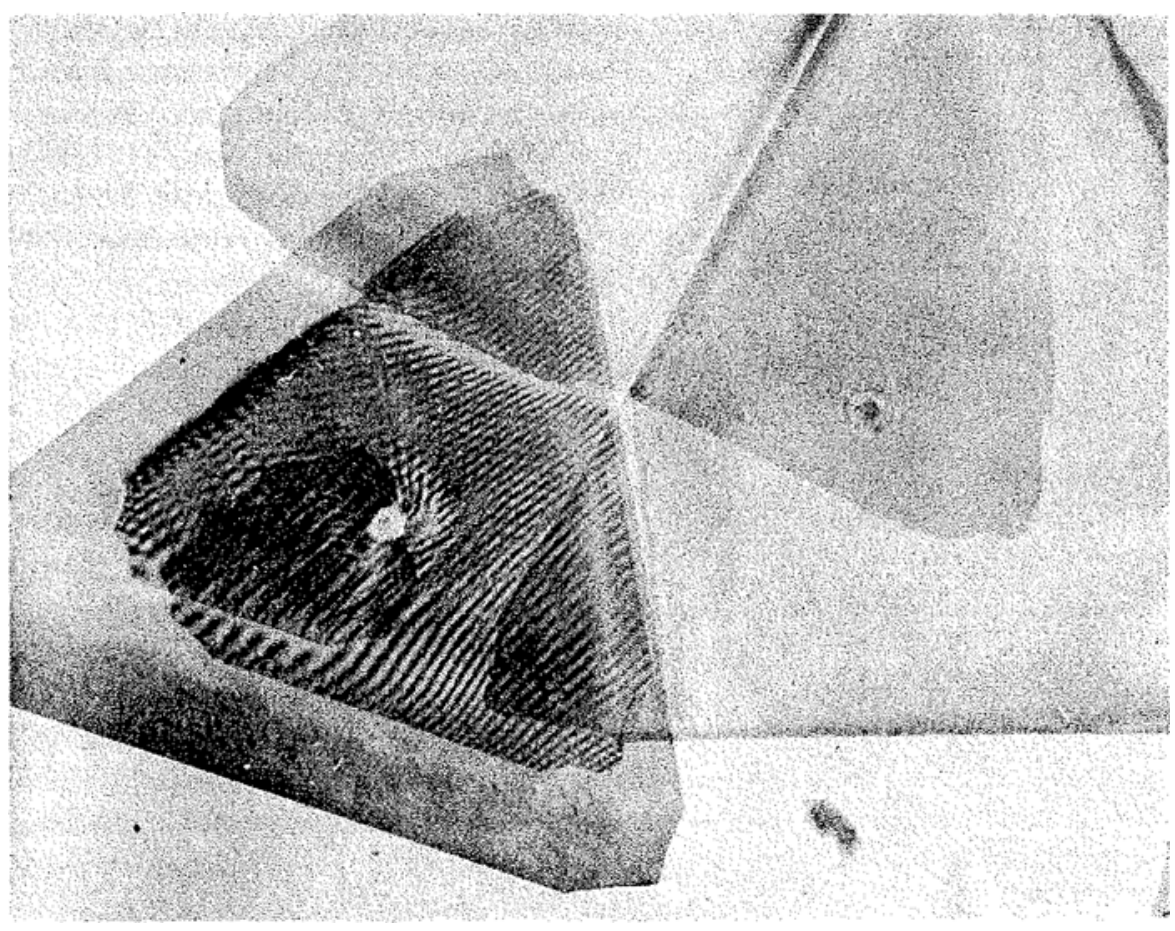

Fig. 4 Plate form of colloidal gold

$x 48500$

Fig. 5 High Resolution Electronumicrograph of a gold 75/platinum 25 per cent alloy particle. Courtesy L.L. Ban

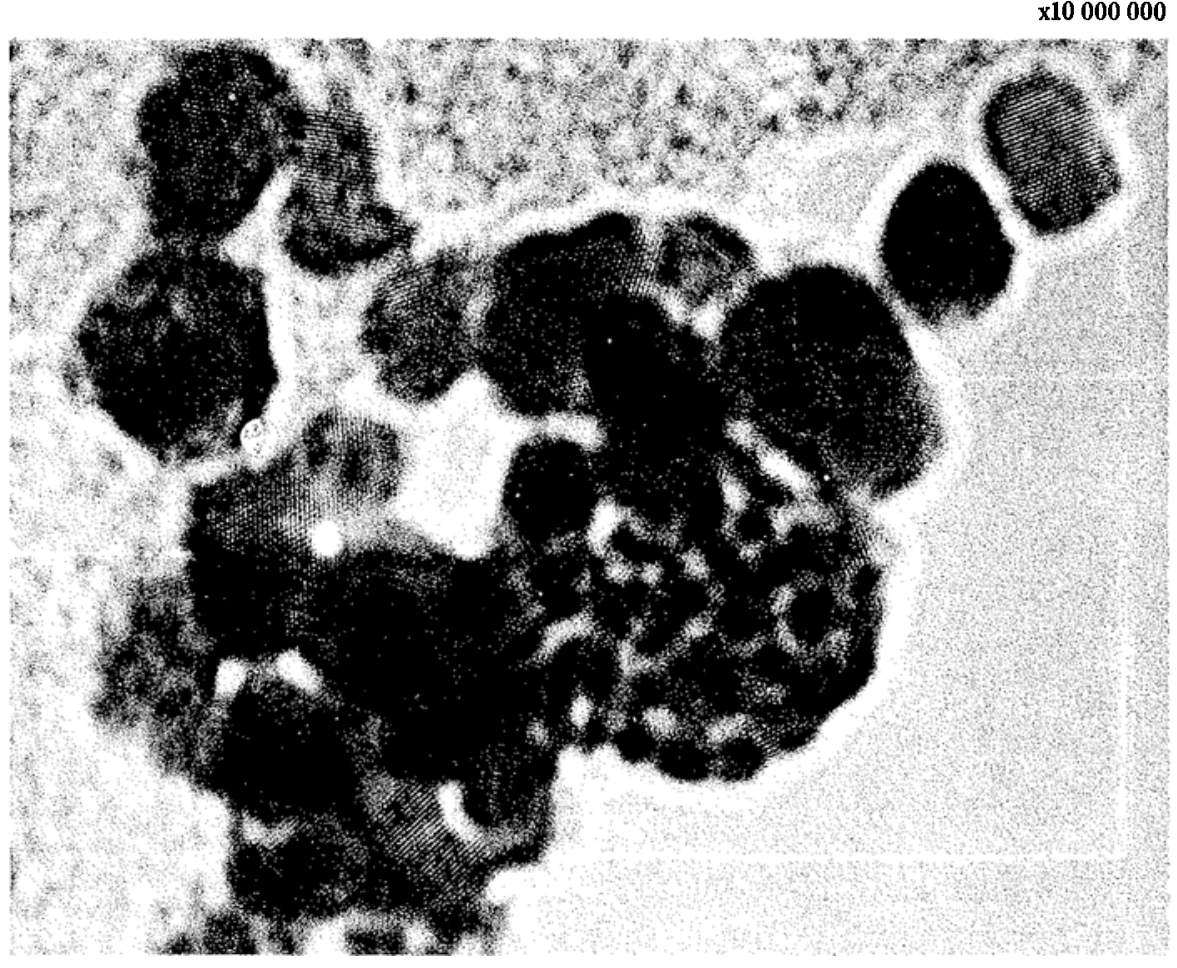

$\mathrm{nm}$ and which were usually spherical in shape and polydisperse in size. As was pointed out above, monodisperse sols can be prepared with an average diameter of 1.4 to $160 \mathrm{~nm}$ and with a mean deviation from the mean of 12 per cent. The particle size distribution is a reflection of the interplay of the nucleation, growth and coagulation processes. The particles may be 'spherical' or plate-like. The 'spherical' particles on examination with an electron microscope of high resolution were found to be icosohedrons with regular faces. They were often twins or multiple aggregates of smaller crystallites. The plates often had five sides. Occasionally the plate-like particleshad a hole through the centre and a growth spiral on each side.

During the last two decades there have been two remarkable developments in electron microscopy: the scanning transmission electron microscope STEM and the high resolution transmission microscope HRTEM (Figure 6).

The STEM has been developed by A.W. Crewe of the University of Chicago and a highly instrumented model was constructed by J.H. Wall of the Brookhaven National Laboratory (25). A point source of high electron intensity is focused by two lenses to produce an electron probe of 0.25 $\mathrm{nm}$ diameter. Appropriate coils scan this electron probe over the specimen. The electrons passing through the specimen are collected by several detectors. The information so obtained is processed and stored on magnetic tape. It can be displayed on a cathode ray tube and also photographed. The resolution issuch that individual heavy atoms such as those of gold can be seen.

It is an annoying situation in electron mictoscopy that when the specimen is in sharp focus and the microscope is at its maximum resolving power, the contrast is very poor. However, for highly stabilized instruments such as the HRTEM and at certain values of the objective lens defocus (controlled to within $10 \mathrm{~nm}$ ) contrast is increased without loss of resolution (26). Particles as small as $0.5 \mathrm{~nm}$ can be visualized 

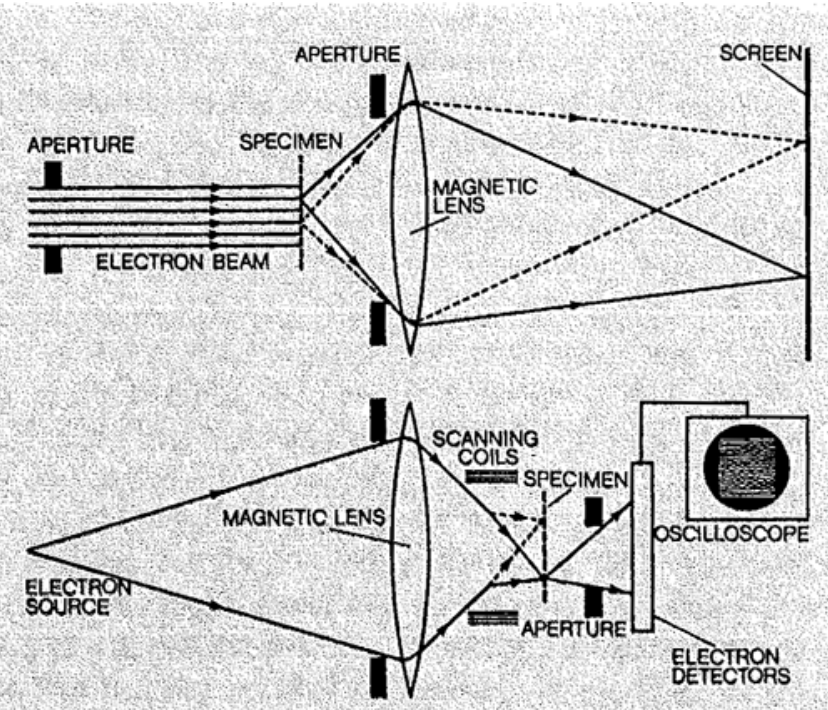

Fig. 6 Operating principles of a High Resolution Transmitting Microscope and a Scanning Transmission Electron Microscope

and lattices of individual particles can be imaged. Variations and dislocations in the lattice planes can be noted. When two gold particles are found close together, a continuity of lattice planes can be seen in the slender bridge between them (Figure 5). A quantitative evaluation of the lattice spacing of an individual particle can be made by a Fourier transform of its electron micrograph. The image of the lattice of the particle made by electrons is used as a diffraction plate for an optical laser beam (Figure 7).

Fig. 7 Principle of an optical diffractometer used for evaluating lattice parametes of individual particles

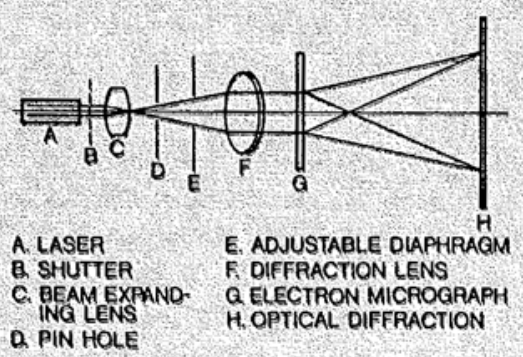

The diffraction spots so obtained (Figure 8) can be measured to determine the lattice parameter of each particle thus examined. Diamond used for calibration gave values varying by $0.01 \mathrm{~nm}$ about its lattice parameter of $2.06 \mathrm{~nm}$. This technique has been used by Ban and the author to obtain distribution curves for lattice parameters of individual particles of colloidal gold-platinum alloys (27). The literature value for $d(111)$ for gold is 2.3499 and for platinum 2.265. The average value for 6 per cent gold alloy was $2.31 \pm 0.06 \AA$ based on 70 particles; for 25 per cent alloy it was $2.32 \pm 0.06 \AA$ based on 87 particles; for 50 per cent alloy it was $2.34 \pm 0.06 \AA$ based on 91 particles; for 75 per cent alloy it was $2.35 \pm 0.05 \AA$ based on 144 particles. A close examination of the distribution curves reveals a small number of particles with $d$ values smaller than those of platinum and a number with $d$ values as high as 2.50. Sedimentation constants were determined using a Beckman ModelE Analytical Centrifuge and these were used to calculate the particle size of the gold-platinum alloys. The diameter was $3.7 \mathrm{~nm}$ for alloys 0 to 50 per cent gold and then it rose linearly with gold composition to a value of $14.5 \mathrm{~nm}$ for 100 per cent gold. The values from electron microscopy were $3.2 \mathrm{~nm}$ for 0 percent, $3.2 \mathrm{~nm}$ for 6.2 per cent, $3.7 \mathrm{~nm}$ for 25 per cent, $4.2 \mathrm{~nm}$ for 50 per cent and $6.0 \mathrm{~nm}$ for 75 per cent gold.

Structure

When a beam of monochromatic X-rays of wavelength $\lambda$ impinges on a material, most of the radiation passes through with a small fraction scattered at a wide angle $\varepsilon$ given by the Bragg relation $n \lambda=2 \mathrm{~d} \sin \theta$, where $\varepsilon=2 \theta$. When the material consists of particles whose diameter is less than $100 \mathrm{~nm}$, two effects are observed: the wide angle lines and the central beam are broadened (low angle $\mathrm{X}$-ray scattering). There is a distinction in the molecular interpretation of these two effects. The broadening of the wide angle lines is due to size and strains of crystallites composing the particles, and crystallite size does not necessarily coincide with particle size. Several decades ago attempts were made to use monodisperse gold to calibrate this X-ray method for size determination with the results from electron microscopy. These were unsuccessful (28) for a reason that is now understood.

High resolution electron microscopy has shown that the particles in standard citrate gold sol are often composed of several crystallites whose size is obviously smaller than the particle size. On the other hand, the small angle $X$-ray scattering of standard $20 \mathrm{~nm}$ gold particles was used by Hubbell and the author $(29,30)$ to confirm the Guinier relationship between the X-ray intensity at various wavelengths, and the scattering angle. The slope of the curve is proportional to the radius of the particle (Figure 9).

Part II of this review on colloidal gold will appeat in the next issue of Gold Bulletin and will discuss the colour, coagulation aspects, adhesion, alloying and catalytic properties of gold colloids. 
Fig. 8 Diffraction pattern obtained from lattice image of a gold $32 /$ platinum 68 per cent alloy particle. Courtesy of L.L. Ban

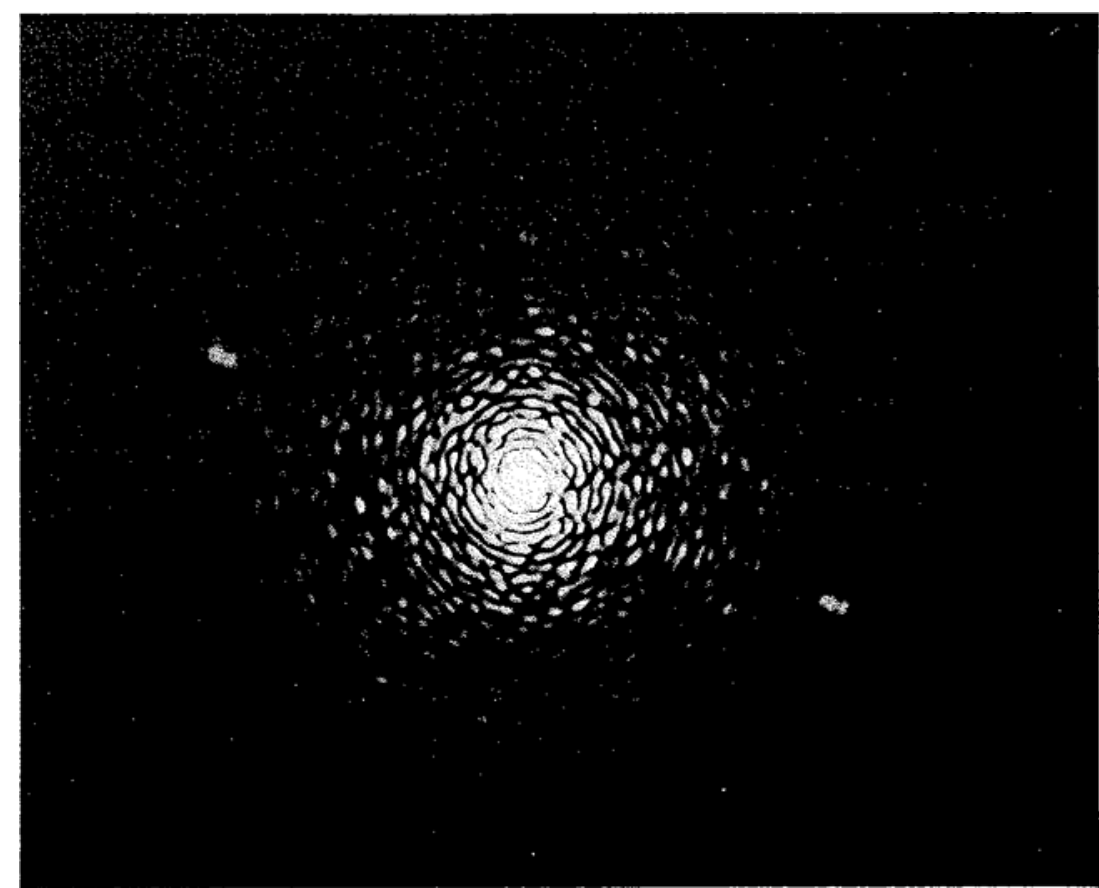

\section{References}

1 R. Zsigmondy and P.A. Thiessen, in 'Das Kolloid Gold', Akad. Verlag, Leipzig, 1925

2 M. Faraday, Pbil. Trans., 1857, 147, 145

3 G. Bredig, Z. ang. Chem., 1898, 11,952

4 R. Zsigmondy, in 'Colloids and the Ultramicroscope',J. Alexander, transl., Wiley, New York, 1909

5 G. Mie, Ann. Pbys. Lpz., 1908, 25, 377

6 H. Schulze, J. Prakt, Chem., 1882, 25, 431

7 W.B. Hardy, Proc. Roy. Soc., 1900, 66, 110

8 A. Einstein, Ann. Phys. Lpz, 1905, 17, 549

9 M. von Smoluchowski, Phys. Z., 1916, 17, 557, 58 s

10 B. von Borries and G.A. Kausche, Kolloid Z., 1940,90, 132

11 M. von Ardenne, Zeit. Physik. Chem. A., 1940, 187, 1

12 J. Turkevich and J. Hillier, Anal. Chem., 1949, 21, 475

13 J. Turkevich, J. Hillier and P.C. Stevenson, Discussions of the Faraday Society No. $11,1951,55-74$

14 K. Takyuama, Bull. Chem. Soc. Japan, 1958, 31, 944

15 N. Uyeda, M. Nishino and E. Suito, J. Coll. Interface Sci. 1973, 43, 264

16 R.H. Morriss and W.O. Milligan, J. Electronmicrosc., 1960, 8, 17

17 C.L. Baignet and G. Muller, Experimentia, 1980, 36, 472

18 K. Kurihara, J. Kizling, P. Stenius and J.H. Fendler, J. Am. Chem. Soc,, 1983, 105,2574

19 F.H. Fry, G.A. Hamilton and J. Turkevich, J. Inorg. Chem., 1966, 5, 1943

20 P.G. Jones, Gold Bull., 1983, 16, 114

21 Y. Chiang and J. Turkevich, J. Coll. Sci., 1963, 18, 772

22 J.A.A. Engelbrecht and H.C. Snyman, Gold Bull., 1983, 16, 66

23 G. Frens, Nature, 1973, 241, 20

24 H. Muehlpfordt, Experientia, 1982, 38, 1127

25 A.V. Crewe, D.M. Eggenburg, J. Wall and L.M. Welter, Rev. Sci. Instr, 1968, 39, 576

26 L.L. Ban, in 'Surface and Defect Properties of Solids' Chemical Society of London, $1972,1,52$

27 R.S. Miner, Jr., S. Namba and J. Turkevich, in 'Proc. 7th Internat. Congr. on Catal.', 1980, Tokyo

$28 \mathrm{~J}$. Turkevich, unpublished results

$29 \mathrm{~J}$. Turkevich, H.H. Hubbell and J. Hiller in 'Discussions of the Faraday Society', $1950,8,348$

30 J. Turkevich and H.H. Hubbell, J. Am. Chem. Soc., 1951, 73, 1

Fig. 9 Small-angle X-ray scattering of standard citrate gold sol

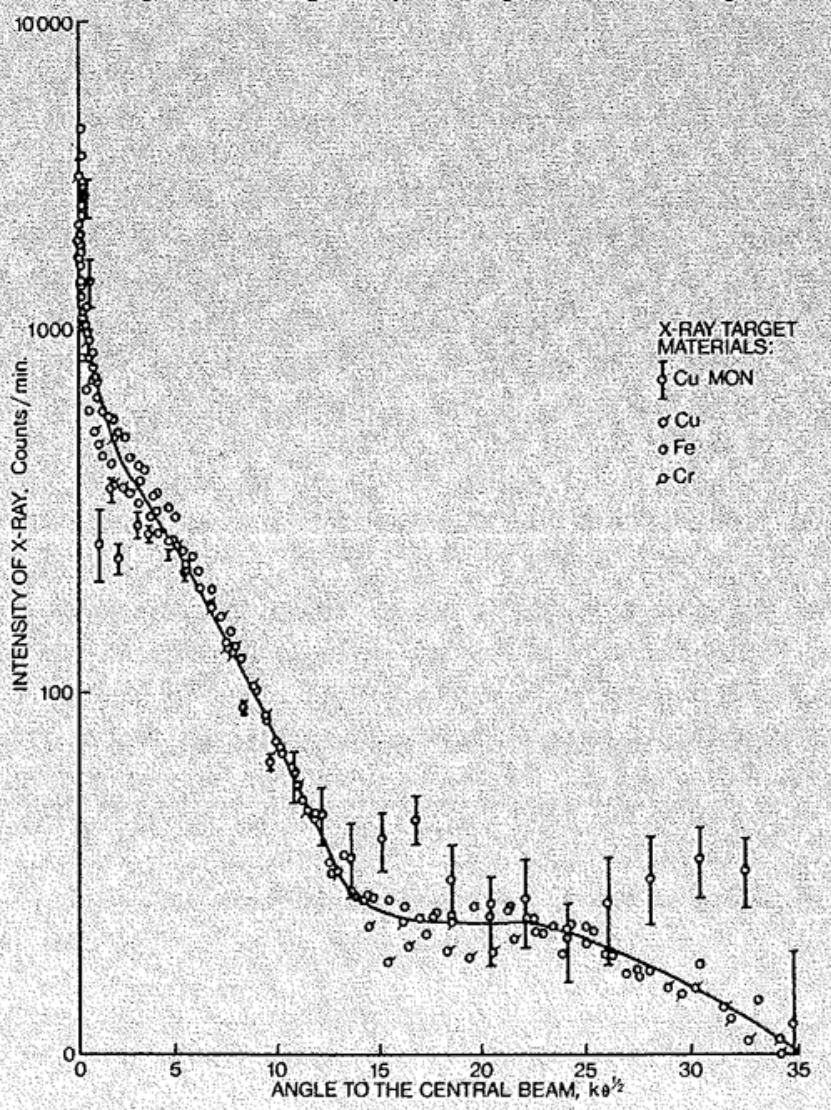

